C2005 IEEE. Personal use of this material is permitted. However, permission to reprint/republish this material for advertising or promotional purposes or for creating new collective works for resale or redistribution to servers or lists, or to reuse any copyrighted component of this work in other works must be obtained from the IEEE. 


\title{
Energy Relaxation Times in a Nb Persistent Current Qubit
}

\author{
Yang Yu, William D. Oliver, D. Nakada, Janice C. Lee, Karl K. Berggren, and T. P. Orlando
}

\begin{abstract}
We measured the energy relaxation time in a $\mathrm{Nb}$ superconducting persistent current qubit using a time-resolved fast measurement scheme. The energy relaxation time is longer than $10 \mu \mathrm{s}$, showing a strong potential of realizing quantum computation with $\mathrm{Nb}$-based superconducting qubits.
\end{abstract}

Index Terms-Nanotechnology, quantum computation, superconducting devices.

\section{INTRODUCTION}

$\mathbf{Q}$ UANTUM computers hold the promise to solve certain problems which cannot be solved by classical computers efficiently [1]. Among those approaches for the development of the quantum computers, use of superconducting qubits (SQs) based on the Josephson devices is promising due to the relative ease of circuit design, fabrication and scaling up [2]-[4]. However, short decoherence times, resulting from the strong coupling between the SQs and the environment, is a common drawback for all superconducting qubits. Although recent progress with the superconducting qubits indicates that long decoherence times are possible [5]-[14], work still needs to be done in order to improve the decoherence time. Recently, we measured the intra-well energy relaxation time in a $\mathrm{Nb}$ superconducting persistent current (PC) qubit [15]. It was found that the intra-well energy relaxation is about $25 \mu \mathrm{s}$, which suggested a long inter-well relaxation time. Here, we present direct time-resolved measurements of the inter-well energy relaxation time between two potential wells, i.e., the energy relaxation time $T_{1}$, in a $\mathrm{Nb} \mathrm{PC}$ qubit. It was found experimentally that $T_{1}>10 \mu \mathrm{s}$. These long energy relaxation times indicate a strong potential for quantum computation employing Nb-based SQs.

A PC qubit is a superconducting loop interrupted by three under-damped Josephson junctions (JJs) (Fig. 1(a)). Two JJs are designed to have the same size and critical current, and the third one is $\alpha$ times smaller in size and critical current. For

Manuscript received October 4, 2004. This work was supported in part by the Air Force Office of Scientific Research (AFOSR) under Grant F49620-01-1-0457 under the DoD University Research Initiative on Nanotechnology (DURINT) Program and by ARDA. The work at MIT Lincoln Laboratory was supported in part by ARDA under Air Force Contract F19628-00-C-0002. The opinions, interpretations, conclusions, and recommendations are those of the author(s) and are not necessarily endorsed by the United States government.

Y. Yu, D. Nakada, J. C. Lee, K. K. Berggren, and T. P. Orlando are with the Massachusetts Institute of Technology, Cambridge, MA 02139 USA (e-mail: yangyu@mit.edu; dnakada@alum.mit.edu; JanLee@mit.edu; Berggren@ mit.edu; Orlando@mit.edu).

W. D. Oliver is with the MIT Lincoln Laboratory, Lexington, MA 02420 USA (e-mail: oliver@1l.mit.edu).

Digital Object Identifier 10.1109/TASC.2005.850078
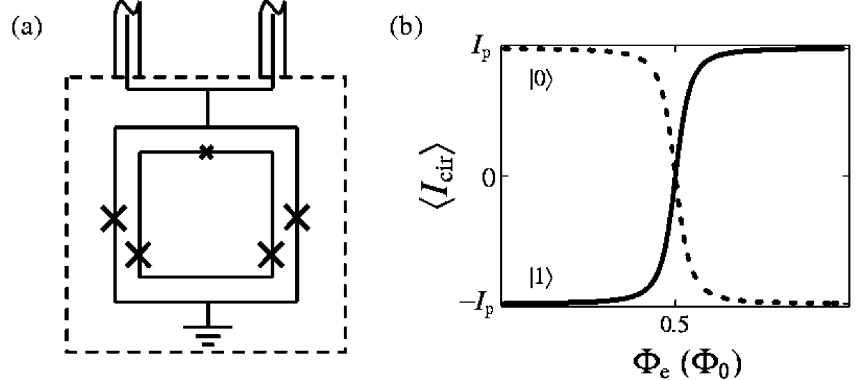

Fig. 1. (a) Schematic of PC qubit and sample package (dashed line). The crosses represent Josephson junctions. The leads of readout SQUID are bonded to the center conductors of the coaxial cables that connected to the room temperature electronics. (b) The quantum mechanical expectation values of the circulating currents in the loop for the qubit ground state (dashed line) and excited state (solid line) as a function of applied magnetic field.

$0.5<\alpha<1$ and with an externally applied magnetic field close to a half flux quantum, $\Phi_{0} / 2$, the system behaves as a particle moving in a double well potential, with the classical states in each well corresponding to macroscopic persistent currents of opposite sign [3]. The potential can be tilted back and forth by changing the frustration $f_{\mathrm{q}}$, which is the magnetic flux threading the loop in units of $\Phi_{0}$. At low temperature and weak damping, the dynamics of the system are governed by quantum mechanics, and the particle occupies quantized energy levels in the double potential well. The two classical states are now coupled via quantum tunneling through the barrier between the wells, and the system behaves as a two level quantum system. Fig. 1(b) shows the expectation values of the circulating persistent currents of the qubit ground state and excited state as a function of the applied magnetic field. The circulating current of the qubit states changes sign with the magnetic frustration passing 0.5 . In addition, the system can interact quantum mechanically with a monochromatic electromagnetic (microwave) field, and microwaves with frequency matching the energy level spacing can generate transitions between the two macroscopic quantum states either coherently or incoherently [6], [13], [15].

\section{EXPERIMENTS AND RESULTS}

The samples used in this study were fabricated at MIT Lincoln Laboratory in a niobium trilayer process [16]. The critical temperature $T_{c}$ of the JJs was $\sim 9 \mathrm{~K}$. The PC qubit area is $16 \times 16 \mu \mathrm{m}^{2}$, with self-inductance of $L_{\mathrm{q}} \simeq 30 \mathrm{pH}$. The critical current density of the junctions is about $180 \mathrm{~A} / \mathrm{cm}^{2}$. The junctions were of high quality, with a sub-gap resistance larger than $1 \mathrm{M} \Omega$. The readout dc SQUID, which surrounds the qubit, consists two JJs that have equal critical current $I_{\mathrm{c} 0} \simeq 2 \mu \mathrm{A}$. Both JJs are shunted with a $1 \mathrm{pF}$ capacitor to lower the resonant 
(a) Microwave
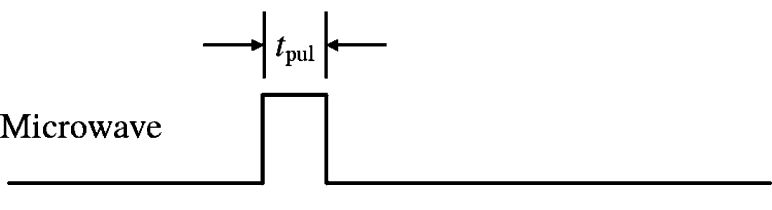

(b) SQUID Current

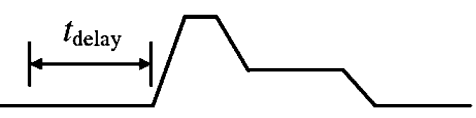

(c) SQUID Voltage

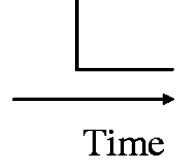

Fig. 2. Time profiles of (a) microwave pulse, (b) SQUID readout pulse and (c) SQUID voltage response for one measurement trial. This procedure was repeated at a $100-1000 \mathrm{~Hz}$ rate to measure the SQUID switching probability. The readout current pulse has a $5 \mathrm{~ns}$ rising time which is not to scale in the plot. In the period of $t_{\text {delay }}$, the microwave was turned off and the qubit performed free evolution.

frequency of the SQUID. The SQUID is $20 \times 20 \mu \mathrm{m}^{2}$ in area, with the self-inductance of $L_{\mathrm{SQ}} \simeq 60 \mathrm{pH}$. The mutual inductance between the qubit and dc SQUID is $M \simeq 25 \mathrm{pH}$. The inductances and $I_{\mathrm{c} 0}$ were determined from the SQUID transfer function measurement and consistent with the estimated values from the process parameters. The persistent current in the qubit will generate an additional magnetic flux of $\sim 5 \mathrm{~m} \Phi_{0}$ in the SQUID, resulting in a $50 \mathrm{nA}$ change on the switching current $I_{\mathrm{sw}}$ of the SQUID which can be easily detected by the SQUID at low temperature. The sample was mounted in a small metal package that was thermally anchored to the mixing chamber of a dilution refrigerator. The devices were magnetically shielded by four cryoperm-10 cylinders surrounding the inner vacuum can, as well as additional superconducting shielding outside the sample package. The readout SQUID was connected to room temperature electronics by semi-rigid coaxial cables, filtered with $40 \mathrm{~dB}$ attenuator and copper powder filter. The bandwidth of the readout setup is about $1 \mathrm{GHz}$, which enabled us to quickly probe the SQUID and readout the qubit states before they are disturbed by the environment. Microwaves can be applied to the qubit through microwave coaxial cables and a loop inductively coupled to the qubit.

The measurement procedure consisted of three steps: initializing, manipulating, and probing. For each measurement trial (Fig. 2), we first initialized the qubit in the ground state $|0\rangle$ by waiting a sufficient long time (typically 1 to $10 \mathrm{~ms}$ ). Then a microwave pulse with duration time $t_{\mathrm{pul}} \sim 0.01$ to $10 \mu \mathrm{s}$ was applied to manipulate the qubit. After the microwaves were turned off, a probing current pulse was sent to the SQUID to read out the qubit state. The current pulse rise time is $5 \mathrm{~ns}$. The current was held constant at $I_{\mathrm{b}}$ for $10 \mathrm{~ns}$, then decreased to $I_{\mathrm{b}} / 2$ and kept there for $t_{\text {hold }}=20 \mu$ s before it was decreased to 0 . For the qubit in the clockwise (counter-clockwise) persistent current state, the SQUID has higher (lower) switching current, the $I_{\mathrm{b}}$ was chosen so that when the qubit is in counter-clockwise (clockwise) persistent current state, the SQUID will (not) switch to voltage state. The switching probability is thus proportional to the population of the qubit in the counter-clockwise persistent current state, or

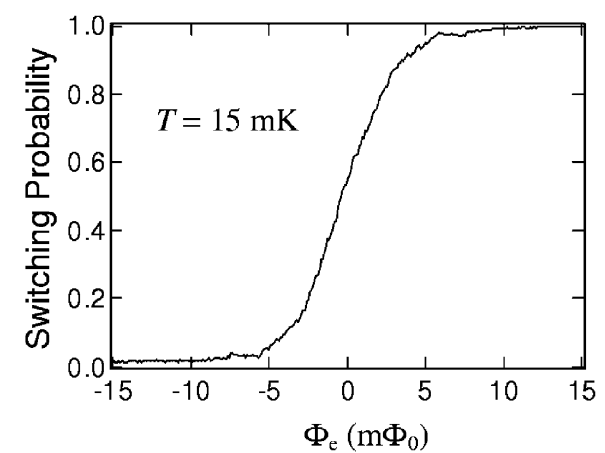

Fig. 3. Switching probability as a function of external magnetic field without microwave irradiation. The qubit was in the ground state $|0\rangle$. The readout persistent current is consistent with the theory calculation in Fig. 1(b).

right well. The voltage across the SQUID was sent to a counter. The $t_{\text {hold }}$ was set for trigging the counter with the highest fidelity. It is worth noting that we did not need to probe the qubit state immediately after turning off the microwave. We let the system freely evolve for a period of time $t_{\text {delay }}$, and the decay of the population of the qubit excited state gives the energy relaxation time. The measurement procedure was repeated a thousand times to get the switching probability with a small statistical error. Fig. 3 shows the measured switching probability as a function of the external magnetic field at the base temperature $(15 \mathrm{mK})$ of our dilution refrigerator. No microwaves were applied; therefore, the qubit was always in ground state $|0\rangle$. For $\Phi_{\mathrm{e}} \ll \Phi_{0} / 2$, the population in the right well is less than $1 \%$ because the energy of the particle in the left well is much lower than that in right well and the system is localized in the left well. With the magnetic field increased, the energy of the right well decreases while that of the left well increases. For $\Phi_{\mathrm{e}} \gg \Phi_{0} / 2$ the right well became energetically preferable and the qubit has a $100 \%$ probability of being in the right well. This plot indicates our readout has fidelity higher than $99 \%$.

We can manipulate the qubit state with a microwave pulse after the qubit state is initialized in the ground state. When the microwave frequency matches the energy separation $\Delta E$ between the ground state and excited state, the qubit can be pumped to the excited state. Fig. 4 shows the qubit population as a function of magnetic field with $\nu=10 \mathrm{GHz}$ microwave irradiation. The pulse width is $t_{\mathrm{pul}}=1 \mu \mathrm{s}$. When the magnetic field was biased at about $5 \mathrm{~m} \Phi_{0}, \Delta E=\nu$, and the qubit was pumped to the excited state. The population in the excited state increased substantially; therefore, a resonant peak (dip) was observed there. If we applied a microwave pulse with a different frequency, the resonant position of the peak and dip moved with the applied magnetic field to satisfy $\Delta E=\nu$. Therefore, by gradually changing the microwave frequency and measuring the corresponding distance of the resonant peak and dip, we can map out the energy band structure of our qubit, as shown in the inset of Fig. 4.

After the microwave irradiation is turned off, the qubit will relax to the ground state in the time scale of the energy relaxation time $T_{1}$. The microwave irradiation pumped the qubit to the excited state at resonant peak. The peak amplitude is proportional to the population on the excited state. Therefore, we can measure $T_{1}$ by measuring the peak amplitude as a function of the probing pulse delay time $t_{\text {delay }}$. Fig. 5 shows an example of 


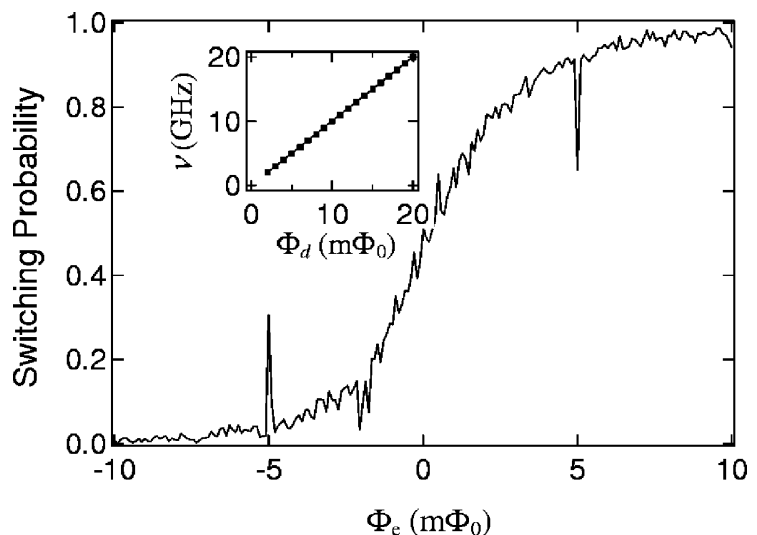

Fig. 4. Switching probability vs. external magnetic field with $10 \mathrm{GHz}$ microwave irradiation. Inset: microwave frequency vs. the distance between the peak and the dip (symbols). The solid line is the theoretical calculation using qubit parameters

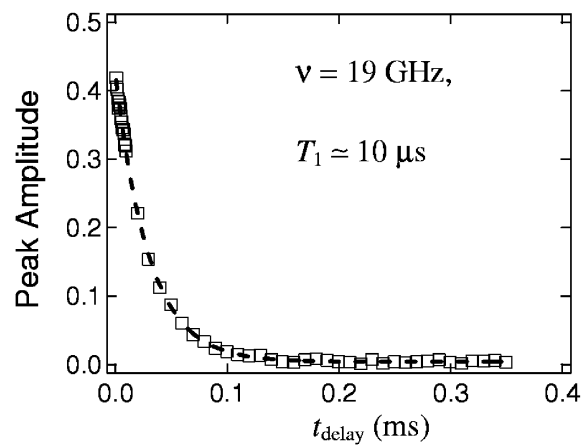

Fig. 5. Normalized resonant peak amplitude as a function of $t_{\text {delay }}$. The dashed line is the best fit to exponential decay with a time constant $T_{1} \simeq 10 \mu \mathrm{s}$. The experimental data show remarkable agreement with the theory prediction.

the resonant peak amplitude as a function of $t_{\text {delay }}$. It exhibits an exponential decay with a time constant $T_{1}$ about $10 \mu \mathrm{s}$. We also measured the $T_{1}$ at other frequencies, ranging from $3 \mathrm{GHz}$ to $20 \mathrm{GHz}$. All energy relaxation times were longer than $10 \mu \mathrm{s}$. From $T_{1}$, we can determine the quality factor of classical small oscillation in the potential well $Q=\Delta E \cdot T_{1} \approx 10^{5}$. These results are consistent with our previous intra-well energy relaxation time measurements as well as dc spectroscopy measurements [15], [17], [18].

\section{DISCUSSION}

This long energy relaxation time is important for experiments in QC in two ways. First, the long energy relaxation time indicates our PC qubit are promising to quantum computing. Because our PC qubit had no leads directly connected to it and the magnetic coupling circuit is optimally designed to lessen the effects of the electromagnetic environment, the PC qubit is much less influenced by this environment than similar singlejunction schemes [10], [11]. Additionally, considering the attractiveness of Nb-based SQs from the point of view of its robust and well-developed fabrication methods, these times indicate that $\mathrm{Nb}$ qubits are promising candidates for realizing a scalable quantum computer. Second, the qubit decoherence time consists of the energy relaxation time $T_{1}$ and the phase relaxation time $T_{2}$. If the energy relaxation is the only decoherence source, $T_{2}$ is twice of $T_{1}$. For an ohmic environment, according to the widely used spin boson model, $T_{1}$ and $T_{2}$ are related to each other from [19], [20],

$$
\begin{aligned}
& T_{1}^{-1} \simeq \frac{\pi \alpha_{L} \sin ^{2} \eta \Delta E}{\hbar}, \\
& T_{2}^{-1}=\frac{T_{1}^{-1}}{2}+\frac{2 \pi \alpha_{L} k_{B} T \cos ^{2} \eta}{\hbar},
\end{aligned}
$$

where $\eta \approx \operatorname{tg}^{-1}(\Delta / \Delta E)$ is the mixing angle, $\Delta$ is the tunneling amplitude between the wells, and $\alpha_{L} \sim 1 / Q$ is the quantum damping parameter [21], [22] which we estimate using our measured $Q$ value. For $\mathrm{Nb}$ PC qubit with a gap $\Delta \approx 2 \mathrm{GHz}$ and operating at $\Delta E \approx 4 \mathrm{GHz}$, a conservative estimate gives $T_{2} \gtrsim 10 \mu \mathrm{s}$ at $15 \mathrm{mK}$. We emphasize that an ohmic environment model may not adequately describe all sources of decoherence; these times must be viewed as estimates pending experimental verification. Nonetheless, for a typical Rabi frequency $\Omega=1 \mathrm{GHz}$, we obtained a quantum quality factor $>10^{4}$, larger than the oft-quoted basic requirement for error-tolerant $\mathrm{QC}$.

\section{CONCLUSION}

In conclusion, we directly measured the energy relaxation time of a Nb-based PC qubit using the time-resolved fast measurement scheme. The energy relaxation time is about $10 \mu \mathrm{s}$ with a system $Q$ factor of greater than $10^{5}$, indicating that our qubit is well-isolated from the electromagnetic environment. Our experiments demonstrated good prospects for well-fabricated $\mathrm{Nb}$ junctions, with its more mature technology, to be used as superconducting qubits.

\section{ACKNOWLEDGMENT}

The authors would like to thank J. Habif for technical assistance. They also thank S. Valenzuela, M. Tinkham, L. Levitov, and M. Vavilov for helpful discussions.

\section{REFERENCES}

[1] M. A. Nielsen and I. L. Chuang, Quantum Computation and Quantum Information: Cambridge, 2000, ch. 1.

[2] M. F. Bocko, A. M. Herr, and M. J. Feldman, "Prospects for quantum coherent computation using superconducting electronics," IEEE Trans. Appl. Supercond., vol. ED-7, pp. 3384-3641, Jun. 1997.

[3] T. P. Orlando, J. E. Mooij, L. Tian, C. H. van der Wal, L. S. Levitov, S. Lloyd, and J. J. Mazo, "Superconducting persistent-current qubit," Phys. Rev. $B$, vol. 60 , pp. $15398-15413$, Dec. 1999.

[4] T. P. Orlando, S. Lloyd, L. S. Levitov, K. K. Berggren, M. J. Feldman, M. F. Bocko, J. E. Mooij, C. J. P. Harmans, and C. H. van der Wal, "Flux-based superconducting qubits for quantum computation," Physica C, vol. 372, pp. 194-200, Aug. 2002.

[5] J. R. Friedman, V. Patel, W. Chen, S. K. Tolpygo, and J. E. Lukens, "Quantum superposition of distinct macroscopic states," Nature, vol. 406, pp. 43-46, Jul. 2000.

[6] C. H. van der Wal, A. C. J. ter Haar, F. K. Wilhelm, R. N. Schouten, C. J. P. M. Harmans, T. P. Orlando, S. Lloyd, and J. E. Mooij, "Quantum superposition of macroscopic persistent-current states," Science, vol. 290, pp. 773-777, Oct. 2000.

[7] Y. Nakamura, Y. A. Pushkin, and J. S. Tsai, "Coherent control of macroscopic quantum states in a single-Cooper-pair box," Nature, vol. 398, pp. 786-788, Apr. 1999.

[8] S. Han, Y. Yu, X. Chu, S. I. Chu, and Z. Wang, "Time-resolved measurement of dissipation-induced decoherence in a Josephson junction," Science, vol. 293, pp. 1457-1459, Aug. 2001

[9] D. Vion, A. Aassime, A. Cottet, P. Joyez, H. Pothier, C. Urbina, D. Esteve, and M. H. Devoret, "Manipulating the quantum state of an electrical circuit," Science, vol. 296, pp. 886-889, May 2002.

[10] Y. Yu, S. Han, X. Chu, S. I. Chu, and Z. Wang, "Coherent temporal oscillations of macroscopic quantum states in a Josephson junction," Science, vol. 296, pp. 889-892, May 2002. 
[11] J. M. Martinis, S. Nam, J. Aumentado, and C. Urbina, "Rabi oscillations in a large Josephson-junction qubit," Phys. Rev. Lett., vol. 89, p. 117 901, Aug. 2002.

[12] Y. A. Pashkin, T. Yamamoto, O. Astafiev, Y. Nakamura, D. V. Averin, and J. S. Tsai, "Quantum oscillations in two coupled charge qubits," Nature, vol. 421, pp. 823-826, Feb. 2003.

[13] I. Chiorescu, Y. Nakamura, C. J. P. M. Harmans, and J. E. Mooij, "Coherent quantum dynamics of a superconducting flux qubit," Science, vol. 299, pp. 1869-1871, Mar. 2003.

[14] A. J. Berkley, H. Xu, R. C. Ramos, M. A. Gubrud, F. W. Strauch, P. R. Johnson, J. R. Anderson, A. J. Dragt, C. J. Lobb, and F. C. Wellstood, "Entangled macroscopic quantum states in two superconducting qubits," Science, vol. 300, pp. 1548-1550, Jun. 2003.

[15] Y. Yu, D. Nakada, J. C. Lee, B. Singh, D. S. Crankshaw, T. P. Orlando, K. K. Berggren, and W. D. Oliver, "Energy relaxation time between macroscopic quantum levels in a superconducting persistent-current qubit," Phys. Rev. Lett., vol. 92, p. 117 904, Mar. 2004.

[16] K. K. Berggren, E. M. Macedo, D. A. Feld, and J. P. Sage, "Low T-c super-conductive circuits fabricated on 150-mm-diameter wafers using a doubly planarized $\mathrm{Nb} / \mathrm{AlOx} / \mathrm{Nb}$ process," IEEE Trans. Appl. Supercond., vol. ED-9, pp. 3271-3274, Jun. 1999.
[17] K. Segall, D. Crankshaw, D. Nakada, T. P. Orlando, L. S. Levitov, S. Lloyd, N. Markovic, S. O. Valenzuela, M. Tinkham, and K. K. Berggren, "Impact of time-ordered measurements of the two states in a niobium superconducting qubit structure," Phys. Rev. B, vol. 67, p. 220 506, Jun. 2003.

[18] D. S. Crankshaw, K. Segall, D. Nakada, T. P. Orlando, L. S. Levitov, S. Lloyd, S. O. Valenzuela, N. Markovic, M. Tinkham, and K. K. Berggren, "Dc measurements of macroscopic quantum levels in a superconducting qubit structure with a time-ordered meter," Phys. Rev. B, vol. 69, pp. 144 518-144 527, Apr. 2004.

[19] A. J. Leggett, S. Chakravarty, A. T. Dorsey, M. P. A. Fisher, A. Garg, and W. Zwerger, "Dynamics of the dissipative two-state system," Rev. Mod. Phys., vol. 59, pp. 1-85, Jan. 1987.

[20] Y. Makhlin, G. Schön, and A. Shnirman, "Quantum-state engineering with Josephson-junction devices," Rev. Mod. Phys., vol. 73, pp. 357-401, 2001.

[21] J. M. Schmidt, A. N. Cleland, and J. Clarke, "Resonant tunneling in small current-biased Josephson junctions," Phys. Rev. B, vol. 43, pp. 229-238, Jan. 1991.

[22] W. Bialek, S. Chakravarty, and S. Kivelson, "Tunneling spectroscopy of a macroscopic variable," Phys. Rev. B, vol. 35, pp. 120-123, Jan. 1987. 